\title{
Elemental analysis in environmental land samples by stand-off laser induced breakdown spectroscopy
}

\author{
Xiao Fang* and S R Ahmad \\ DEAS, CDS, Cranfield University, Shrivenham, SN6 8LA, UK \\ * Corresponding author: x.fang@cranfield.ac.uk
}

\begin{abstract}
The stand-off detection and analysis of environmental land samples have been demonstrated using laser induced breakdown spectrometry. The samples of interest have included soils and vegetation powder. Elements $\mathrm{Hg}, \mathrm{As}, \mathrm{Pb}, \mathrm{Zn}, \mathrm{Cd}$ and $\mathrm{Cr}$ have been spectrally analysed with a focus on $\mathrm{Hg}$ as a trace contaminant in the samples. It is found that element $\mathrm{Fe}$, usually contained in land samples, is a main source of spectral interference for $\mathrm{Hg}$ detection, due to its ever present Iron emission line at $253.68 \mathrm{~nm}$ that is closely adjacent to the strongest $\mathrm{Hg}$ emission line at $253.65 \mathrm{~nm}$, and hence a high resolution of spectral detection is necessary. The strong spectral signals from Bremsstrahlung emission in laser induced plasma and atomic emission of $\mathrm{Fe}$ of high concentration caused a significant reduction of detection resolution in the use of image intensifier of an ICCD. The limit of detection at $\sim 8 \mathrm{ppm}$ for $\mathrm{Hg}$ detection in soil samples with iron as a minor constituent has been achieved, using an optical chopper and a CCD detector for laser induced breakdown spectroscopy (LIBS) signal detection. Such detection method in LIBS system has shown a great advantage in determining trace elements from interfering elemental constituents in land sample matrixes.
\end{abstract}

\section{INTRODUCTION}

Laser-induced breakdown spectroscopy (LIBS) as a viable analytical tool has been applied to elemental detection and analysis in a wide range of analytical fields in the last decade [1 - 10], and it has attracted increasing attention from both the scientific and commercial communities. The laser used in this technique as an atomization and ionization tool offers some major advantages over the conventional atomization or ionization devices in atomic spectroscopy. In particular, it allows in situ, real-time and stand-off detection/monitoring without the need for any sample preparation protocol. The major emphasis in the development of LIBS system has so far been focused on its use with solid samples, culminating in commercial systems for remote and in situ elemental analysis for hazardous and inaccessible targets [11 - 15]. One of the most important applications of LIBS is the determination of trace elements in contaminated land for environmental monitoring and waste management, particularly when some locations are inaccessible or sample targets are in hostile environments.

The pollution of land through soil and plants is a big threat to the environment. Among those pollutants, heavy metals are often present as a consequence of anthropogenic activities (Intensified industrialisation and human activities) and are also the most concerned polluting elements due to their toxicity and their susceptibility to bioaccumulation. There have been numerous investigations and application demonstrations reported by world-wide researchers and technologists using LIBS technique for soil and plant analysis [16 - 22]. In the present state of this development, LIBS cannot compete with the established analysis methods, such as Inductively Coupled Plasma-Atomic Emission Spectroscopy (ICP-AES), Gas Chromatography Mass Spectroscopy (GCMS) etc., in terms of limits of detection and reproducibility. However, these methods need the use of trained personnel and long time for 
sample preparation and presentation for extracting elements from solid samples and making the sample solution prior to measurements. Therefore, LIBS has its own great role to play in rapid elemental analysis of solid samples.

The experimental study for LIBS in soils and plants has been carried out in our laboratory and showed that for the monitoring of some priority elements such as $\mathrm{Hg}, \mathrm{As}, \mathrm{Pb}$ etc. in soils the detection resolution and sensitivity need to be further improved. Spectral interferences from the elements contained in the sample matrixes have also caused main difficulties for identifying trace contaminants from the minor or major elemental constituents. Iron, an element that is naturally and mostly present as a minor constituent in land environmental samples, produces a great spectral interference in trace mercury detection due to $\mathrm{Fe}$ emission line at $253.68 \mathrm{~nm}$ being close to that of $\mathrm{Hg}$ at $253.65 \mathrm{~nm}$. This paper presents the experimental results and analysis on the trace detection of heavy metals, e.g. mercury, etc. in soil and plant samples. The detection limit for mercury has been clearly improved when a high resolution Echelle grating spectrograph coupled with CCD and an optical chopper as a gating device was used, indicative of the prospect of its commercial viability. These results were compared with those using an LIBS system with ICCD which may have a significant effect on spectral detection resolution, restricting the utilisation of the high resolution of a spectrograph.

\section{MATERIALS AND METHODS}

\subsection{Samples}

A selection of environmental samples of soil and plant were used for this experimental study. The soil materials were Clay Soil (LGCQC3004, LGC Promochem, UK), and the plant material was dry powder of tomato leaves (locally grown) in which As and $\mathrm{Hg}$ were added at a trace concentration (50 ppm) for detection purpose. An archived soil material (Cranfield Natural Resources, UK) was also doped with trace mercury at a set of various concentrations and used for generating $\mathrm{Hg}$ concentration curve. The contents of the elements of interest for detection in the clay soil and plant samples are listed in Table 1. All samples were pressed into $\sim 10 \mathrm{~mm}$ diameter disc of $2 \mathrm{~mm}$ thickness under a pressing pressure of $\sim 15$ tons on each dry sample. Sample discs were placed on the sample translational device allowing data collection from a fresh surface for each laser pulse, thereby reducing data averaging error.

\subsection{Experimental Apparatus}

A laboratory based experimental apparatus was set up to carry out initial laser induced breakdown spectrometry. As shown schematically in Fig. 1, it consisted of a laser source, a sample translation stage, a double-grating monochromator (SPEX 1404) and a detection system which is intensified diode array detector connected to an optical multichannel analyser (EG\&G 1420/OMA III). The spectral dispersion is achieved by the monochromator with a maximum resolution of $10 \mathrm{~cm}^{-1}$, as quoted by the manufacturer, which allows a spectral resolution of $<0.063 \mathrm{~nm}$ at $250 \mathrm{~nm}$. The excitation source was an Nd-YAG laser (Litron 200UK), operating at $1064 \mathrm{~nm}$ wavelength $(\lambda)$ and delivering a maximum energy of $200 \mathrm{~mJ}$ over pulse duration of $\sim 6 \mathrm{~ns}(\mathrm{FWHM})$ at a maximum pulse repetition rate of $20 \mathrm{~Hz}$. The beam diameter (D) at the exit was $5 \mathrm{~mm}$ and the beam divergence was $<2 \mathrm{mrad}$. A $100 \mathrm{~mm}$ focal length (f) lens was used to focus the beam, being delivered through a mirror and a prism, on to a sample pellet whose surface was placed in the focal plane of the lens. The radium ( $r$ ) of the laser focus hitting the sample surface was approximately estimated as $26 \mu \mathrm{m}$ using diffraction-limited imaging, i.e. $r=1.22 \lambda f / D$. The laser pulse energy and pulse repetition rate were maintained at $\sim 100 \mathrm{~mJ}$ and $20 \mathrm{~Hz}$ respectively for the present experiments. The spectral 
coverage extended from UV to near IR wavelengths depending upon the grating type being used. The plasma source induced with the exciting laser on the sample surface was imaged on to the entrance slit of the monochromator by both F/2 and F/8 lenses of $50 \mathrm{~mm}$ diameters, giving an $\mathrm{f \#}$ nearly matching that (f/7.8) of the monochromator. The gating on the detector intensifier was controlled by the external trigging pulses in synchronisation with laser output. The system parameters have been optimised for maximum signal detection with reasonable spectral resolutions and noise filtering by gating. A one-dimension translation sample stage was integrated into the system, which allows sampling fresh sample points for spatial averaging of spectral signals.

\section{RESULTS and ANALYSIS}

\subsection{LIBS of tomato leaves}

The spectral measurements and analysis were carried out to evaluate their limits of detection based on the intensities of their spectral peaks and background noises. In all spectral recording and measurements, gate delay and width were optimally set as $2 \mu \mathrm{s}$ and $5 \mu \mathrm{s}$ respectively. Each spectrum was acquired with 200 shots signal averaging at the pulse repetition rate of $20 \mathrm{~Hz}$ to achieve good signal-to-noise ratio $(\mathrm{S} / \mathrm{N})$. The region of interests for LIBS spectral detection was focused on those of prominent emission lines of elements contained in the samples. Using the experimental apparatus of Fig.1, LIBS spectra of these elements in the sample of tomato leave were recorded and examples of these are shown in Fig. 2. The trace elements Zn (62 ppm), Cd (3 ppm), As (50 ppm) Pb (6.3 ppm) and $\mathrm{Cr}(4.5$ ppm) were clearly detected at their strongest emission lines as shown in the spectra. However, the emission line of $\mathrm{Hg}(50 \mathrm{ppm})$ at $253.65 \mathrm{~nm}$ appeared to be interfered with those of $\mathrm{Fe}$ at $253.68 \mathrm{~nm}$ and $\mathrm{P}$ at $253.56 \mathrm{~nm}$. The lines of $\mathrm{Hg}$ and Fe were not resolved although their overlapped signal was detected. These spectral interferences have given rise to an uncertainty for mercury identification and made $\mathrm{Hg}$ undetectable in this measurement.

For the spectra in Fig.2, the signal-to-noise ratios $(\mathrm{S} / \mathrm{N})$ were measured at the chosen emission line of each element, and the limits of detection (LOD) were estimated as $3 \mathrm{C} / \mathrm{S} / \mathrm{N}$, where $C$ is elemental concentration in the sample. This estimation was based on the assumptions of a linear concentration calibration and no spectral interference. Such LOD estimation may not be evidently biased in the cases of $\mathrm{Zn}, \mathrm{Pb}, \mathrm{Cr}, \mathrm{Cd}$ and $\mathrm{As}$, where the spectral interferences were weak and the signals (S) were measured from the local background at non-interference side. The limits of detection for these elements are listed in Table 2 when the LIBS system in Fig. 1 was used. Such detection sensitivities for most of these elements can reach the concentration levels of sub-ppm or ppm which may meet the requirement of minimum permissible limits (MPL) for disposal. This has demonstrated that the LIBS system is viable tool for the detection of these elements. However, not all of the elements in the sample of tomato leaves can be detected with the same level of sensitivities. For example, arsenic and mercury was found to be not amenable to detection with the sensitivity meeting the MPL with the existing LIBS system.

\subsection{LIBS of clay soil}

To observe and analyse the effect of sample matrix containing minor concentrations of constituent, iron, on the detection of mercury and arsenic, LIBS spectra of the certificated Clay Soil sample (LGCQC3004, LGC) with high concentrations of $\mathrm{Hg}$ at $670 \mathrm{ppm}$, As at 83 ppm and minor iron at $4 \%$ were recorded at the regions of emission lines $234.98 \mathrm{~nm}$ for As and $253.65 \mathrm{~nm}$ for $\mathrm{Hg}$, as shown in Fig. 3. Element As was evidently detected on the shoulder 
of spectral signal of iron centred at $234.83 \mathrm{~nm}$ although this emission line of iron produced serve interference. However, the $\mathrm{Hg}$ line could not be resolved from the Fe interfering lines at $253.68 \mathrm{~nm}$, due to insufficient spectral detection resolution. This could be due to insufficient resolution of the spectrograph and also the over exposure of strong Bremsstrahlung emission of continuous spectrum on the photocathode of the image intensifier for the detector. The light over-exposure produces a strong photo-electron noise and thus the photocathode could not sufficiently recover before the intensifying gate is opened. Therefore, it is evident that the existence of iron at a relatively high concentration in environmental sample matrix has made it difficult particularly to detect mercury and demands a more dedicated system of high measurement resolution.

\subsection{High resolution LIBS detection of $\mathrm{Hg}$}

For the detection of mercury in a sample matrix containing minor constituent of iron, two factors were considered in the LIBS system for this study: use of a higher resolution spectrograph and blocking of Bremsstrahlung emission. Therefore, two same Echelle grating spectrographs (ARYELLE Butterfly, Lasertechnik Berlin $\mathrm{GmbH}$ ) were used. One of these was coupled with ICCD as a gateable detector and the other coupled with CCD as a detector and a mechanical chopper as a physical gating device in front of its entrance slit. The spectrograph is of spectral resolving power of $\sim 30,000$ (as quoted by the manufacturer), giving a spectral resolution $\Delta \lambda=\lambda / 30000 \approx 0.008 \mathrm{~nm}$ at the wavelength $(\lambda)$ of $250 \mathrm{~nm}$. Using these two different detection techniques with same LIBS parameter settings, the LIBS spectra of pressed samples of clay soil and tomato leave powder were recorded over 100 shots averaging and their regions of interest at $253.65 \mathrm{~nm}$ are shown in Fig. 4 and Fig. 5 respectively. The gating delay was set as $2 \mu \mathrm{s}$ for both the ICCD and the optical chopper with respect to the laser pulse.

It is shown that spectral signals of the emission line at $253.56 \mathrm{~nm}$ of phosphorus, as a minor constituent in both of the samples, are clearly away from those around $253.65 \mathrm{~nm}$ and thus the existence of phosphor may not affect the detection of mercury when a high resolution spectrograph is used. However, using ICCD detector the spectral lines of $\mathrm{Hg}$ at $253.65 \mathrm{~nm}$ and $\mathrm{Fe}$ at $253.68 \mathrm{~nm}$ were not resolved, as shown in Fig. $4 \mathrm{a}$ and Fig.5a, in spite of the apparently sufficient high resolution $(\sim 0.008 \mathrm{~nm})$ of the used spectrographs. Mercury was not detected in this case due to the interfering line of iron contained in the samples. When CCD detector and a chopper were used, LIBS spectra in both Fig. 4b and Fig. 5b evidently show that the $\mathrm{Hg}$ line from both of the samples has been identified and resolved from an interfering spectral line of iron at $253.68 \mathrm{~nm}$. For tomato leave sample, the Fe line was better resolved because of its lower concentration. In the clay soil sample, iron concentration was much higher $(4 \%)$ than that $(0.07 \%)$ in the sample of tomato leaves and thus its emission line at $253.68 \mathrm{~nm}$ caused worse interference to its nearby $\mathrm{Hg}$ line of $253.65 \mathrm{~nm}$. From the signal-tonoise ratio measurements of these two spectral signals acquired with CCD and the chopper, the limits of detection for mercury were estimated as $\sim 50 \mathrm{ppm}$ in clay soil and $\sim 18 \mathrm{ppm}$ in tomato leaves.

Strong Bremsstrahlung emission of continuous spectrum in laser induced breakdown spectrometry takes place prior to atomic emission of elements in their samples. In use of ICCD, such strong light directly strikes onto the photocathode of its image intensifier and generates massive photon-electrons locally as background noise. The photocathode may not recover before receiving the following spectral signal of atomic emission in a few microseconds. Thus the remaining shot noise is also intensified together with desired spectral 
signal during gated detection. Moreover, in the case of high concentration of iron contained in the samples, the strong spectral signal of Fe emission may cause over-exposure of its amplified electronic signal on to the phosphor screen of image intensifier and consequently reduce resolving capability of the detector. In use of a CCD and a chopper for gating, this effect is avoided, and the strong bremsstrahlung emission is physically blocked from entering the detector to greatly reduce or eliminate the shot noise. This allows the CCD still be able to detect the weak spectral signal from trace mercury with good signal-to-noise ratio, without use of an image intensifier which compromises the utilisation of an ultra-high resolution spectrograph. Therefore, the detection of trace mercury in soil and plant sample matrixes containing minor iron can be achieved with this detection technique.

\subsection{Calibration curve for $\mathrm{Hg}$ detection}

As a result from the above analyses, an ultra-high resolution spectrograph connected with a CCD detector and an optical chopper as a gating device is able to undertake LIBS measurement of trace mercury in its contaminated land samples, where one of the minor constituents, iron usually poses strong spectral interference. To further evaluate the application using this system, concentration calibration for $\mathrm{Hg}$ contained in soil was carried out. The soil (provided by Cranfield Natural Resources, UK) was used as a sample matrix containing $\sim 0.08 \mathrm{ppm}$ of mercury and $\sim 1 \%$ of iron. Mercury of different concentrations in aqueous solutions was added to the matrix samples, which were then dried in a vacuum oven at a temperature of $50^{\circ} \mathrm{C}$ and pressed into pellets. The mercury concentrations of these samples were 0.08 ppm, 25 ppm, 50 ppm, 100 ppm, 200 ppm and 400 ppm. Their LIBS spectra were acquired over 1000 shots averaging at $20 \mathrm{~Hz}$ pulse repetition rate of exciting laser. Fig. 6 shows the spectra around $253.65 \mathrm{~nm} \mathrm{Hg}$ emission line for $\mathrm{Hg}$ concentrations of $0.08 \mathrm{ppm}, 50 \mathrm{ppm}$ and $200 \mathrm{ppm}$. All the spectra are normalised to signal intensity of Fe line at $253.68 \mathrm{~nm}$ and their peak intensities were measured as their peak values subtracting the local background and marked in Fig. 7. A linear calibration curve of lower $(<100 \mathrm{ppm}) \mathrm{Hg}$ concentrations (the first four data points) was also plotted in this figure, with its extension (dot line) to show a strong deviation of the data points at higher $\mathrm{Hg}$ concentrations (>100 ppm). The deviation may indicate that at higher concentrations of $\mathrm{Hg}$, the laser induced plasma is of higher electron density and the plasma absorption (self-absorption) of emission signal increases proportionately, leading to a non-linear effect. Therefore, a linear calibration curve only applies to the case of lower concentration. Based on the slop ( $\rho)$ of this curve, 0.0029 , and measured standard deviation $(\sigma)$ of noise, $\sim 0.0053$, the limit of detection (LOD $=3 \sigma / \rho$ ) was estimated to be $\sim 6 \mathrm{ppm}$. This also shows a great improvement with more shots averaging on spectral acquisition for weak spectral signal of trace elements, such as mercury, using CCD detector.

\section{CONCLUSIONS}

Laser induced breakdown spectroscopy (LIBS) is a feasible technique for stand-off detection and analysis of trace elements in contaminated land samples, e.g. soils and tomato leaves in this study. The elemental constituents of sample matrixes may pose strong spectral interference within a spectral range of the resolution limit. The results clearly indicated that the LOD for the detection of $\mathrm{Hg}$ at its emission line of $253.65 \mathrm{~nm}$ was governed by the interference effect of the ever present Fe line at $253.68 \mathrm{~nm}$. The high concentration of $\mathrm{Fe}$ usually contained in land samples makes mercury detection a very challenging task. Therefore, an ultra-high resolution in spectral detection of a LIBS system is required to take this challenge. The use of image intensifier in ICCD significantly reduced the overall 
performance in spectral resolution, due to the exposure to strong Bremsstrahlung emission and Fe emission signals. This problem can most effectively be addressed by uses of a CCD and an optical chopper to physically block the strong light from Bremsstrahlung emission and also to avoid over-multiplication of emission signal from interfering elements. The detection and analysis of trace elements including mercury in an iron-reach land environmental sample can be achieved at ppm concentration levels using this detection technique with an ultra-high resolution spectrograph. For practical applications, further work is being considered to determine the lower limit of laser pulse energy when using the CCD as compared to the ICCD and also to understand how well the signal is modulated by the optical chopper.

\section{REFERENCES}

1. F. Anabitarte, A. Cobo, J. M. Lopez-Higuera: ISRN Spectroscopy, 285240, 1 (2012)

2. P.K. Diwakar, K.H. Loper, A. Matiaske, D.W. Hahn: J. of Analytical Atomic Spectrometry 27, $1110(2012)$

3. L. Huang, M. Yao, Y. Xu, M. Liu: Applied Physics B: Lasers and Optics, 111, 45 (2013)

4. V. K. Unnikrishnan, R. Nayak, K. Aithal, V. B. Kartha, C. Santhosh, G. P. Gupta, B. M. Suri: Analytical Methods 5, 1294 (2013)

5. M.A. Khater: Spectrochimica Acta, Part B: Atomic Spectroscopy 81, 1 (2013)

6. M.Z. Martin, S. Allman, D.J. Brice, R.C. Martin, N.O. Andre: Spectrochimica Acta, Part B: Atomic Spectroscopy, 74-75, 177 (2012)

7. A.F.M.Y. Haider, R.S. Lubna, K. M. Abedin: Applied Spectroscopy 66, 420 (2012)

8. X. Fang, S. R. Ahmad, M. Mayo, S. Iqbal: Lasers in Medical Science 20, 132 (2005)

9. A. Giacomo, M. Dell'Aglio, A. Casavola, G. Colonna, O. Pascale, M. Capitelli: Analytical and Bioanalytical Chemistry, 385, 303 (2006)

10. X. Fang, S. R. Ahmad: Environmental Technology 26, 1271 (2005)

11. F. J. Fortes, J. J. Laserna: Spectrochimica Acta, Part B: Atomic Spectroscopy 65B, 975 (2010),

12. F.C. De Lucia Jr., A.C. Samuels, R.S. Harmon, R.A. Walters, K.L. McNesby, A. LaPointe, R.J. Winkel Jr., A.W. Miziolek: IEEE Sensors Journal, 5, 681 (2005)

13. M. A. Gondal, T. Hussain, Z. H. Yamani, M. A. Baig: Journal of Hazardous Materials, 163, 1265 (2009)

14. L. Peng, D. Sun, M. Su, J. Han, C. Dong: Optics \& Laser Technology 44, 2469 (2012)

15. M.A. Gondal, M.A. Dastageer, A.A. Naqi, A.A. Isab, Y.W. Maganda: Applied Optics, 51, 7395 (2012)

16. J. El Haddad, M. Villot-Kadri, A. Ismael, G. Gallou, K. Michel, D. Bruyere, V. Laperche, L. Canioni, B. Bousquet: Spectrochimica Acta, Part B: Atomic Spectroscopy 79-80, 51 (2013)

17. J. Kwak, K. Kim, M. Park, J. Kim, K. Park: Environmental Technology, 33, 2177 (2012)

18. D. Santos Jr., L. C. Nunes, G.G.A. de Carvalho, M. da S. Gomes, P.F. de Souza, F. de O. Leme, L.G.C. dos Santos, F.J. Krug: Spectrochimica Acta Part B: Atomic Spectroscopy, 71-72, 3 (2012)

19. Q. Peng, M. Liu, M. Yao, T. Chen: Proceedings of SPIE, 8205, 82050G/1 (2011)

20. O. T. Butler, W. Cairns, J.M. Cook, C.M. Davidson: J. Anal. At. Spectrom., 26, 250 (2011)

21. A.K. Pathak, R. Kumar, V.K. Singh, R. Agrawal, S. Rai, A.K. Rai: Applied Spectroscopy Reviews, 47, 14 (2012)

22. G.G.A. de Carvalho, D. Santos Jr., L.C. Nunes, M. da S. Gomes, F. de O. Leme, F.J. Krug: Spectrochimica Acta Part B: Atomic Spectroscopy, 74-75, 162 (2012) 


\section{Captions}

Table 1 Known elemental concentrations of reference samples

Table 2 Estimated LODs for the chosen elements in tomato leave samples

Fig. 1 Schematic of laboratory apparatus for LIBS stand-off measurement

Fig. 2 LIBS spectra of Tomato Leaves at the emission lines of $\mathrm{Zn}, \mathrm{Cd}, \mathrm{As}, \mathrm{Hg}, \mathrm{Pb}$ and $\mathrm{Cr}$

Fig. 3 LIBS spectra of clay soil at the strong emission lines of As and $\mathrm{Hg}$

Fig. 4 LIBS spectra of clay soil for Hg detection using: a) ICCD and b) CCD with a chopper

Fig. 5 LIBS spectra of tomato leave for $\mathrm{Hg}$ detection, using: a) ICCD detector and b) CCD detector with a chopper

Fig. 6 LIBS spectra of soil samples at $\mathrm{Hg}$ concentrations of a) $0.08 \mathrm{ppm}$, b) $50 \mathrm{ppm}$ and c) $200 \mathrm{ppm}$

Fig. 7 LIBS peak intensity of $\mathrm{Hg}$ at $253.65 \mathrm{~nm}$ versus its concentrations

\section{Tables and Figures}

Table 1

\begin{tabular}{|l|l|l|l|lllllll|}
\hline \multicolumn{2}{|l|}{ Trace elements } & $\mathrm{P}$ & $\mathrm{Fe}$ & $\mathrm{Zn}$ & $\mathrm{Pb}$ & $\mathrm{Cr}$ & $\mathrm{As}$ & $\mathrm{Cd}$ & $\mathrm{Hg}$ \\
\hline \multirow{3}{*}{$\begin{array}{l}\text { Concentration } \\
(\mathrm{ppm})\end{array}$} & Tomato Leaves & $3.4 \mathrm{~K}$ & 690 & 62 & 6.3 & 4.5 & 50 & 3 & 50 \\
\cline { 2 - 9 } & Clay Soil & $40 \mathrm{~K}$ & $40 \mathrm{~K}$ & 82 & 50 & 37 & 83 & 1 & 670 \\
\hline
\end{tabular}

Table 2

\begin{tabular}{|l|l|l|l|l|l|l|}
\hline Trace elements & $\mathbf{Z n}$ & $\mathbf{P b}$ & $\mathbf{C r}$ & $\mathbf{C d}$ & As & Hg \\
\hline $\begin{array}{l}\text { Elemental concentration } \\
\text { (ppm) }\end{array}$ & 62 & 6.3 & 4.5 & 3 & 50 & 50 \\
\hline Emission lines (nm) & 213.86 & 405.78 & 425.43 & 228.8 & 234.98 & 253.65 \\
\hline Limits of detection (ppm) & 2.8 & 1.3 & 0.5 & 1.6 & 20 & * \\
\hline
\end{tabular}

* Spectral signal of $\mathrm{Hg}$ could not be resolved from Fe line interference. 


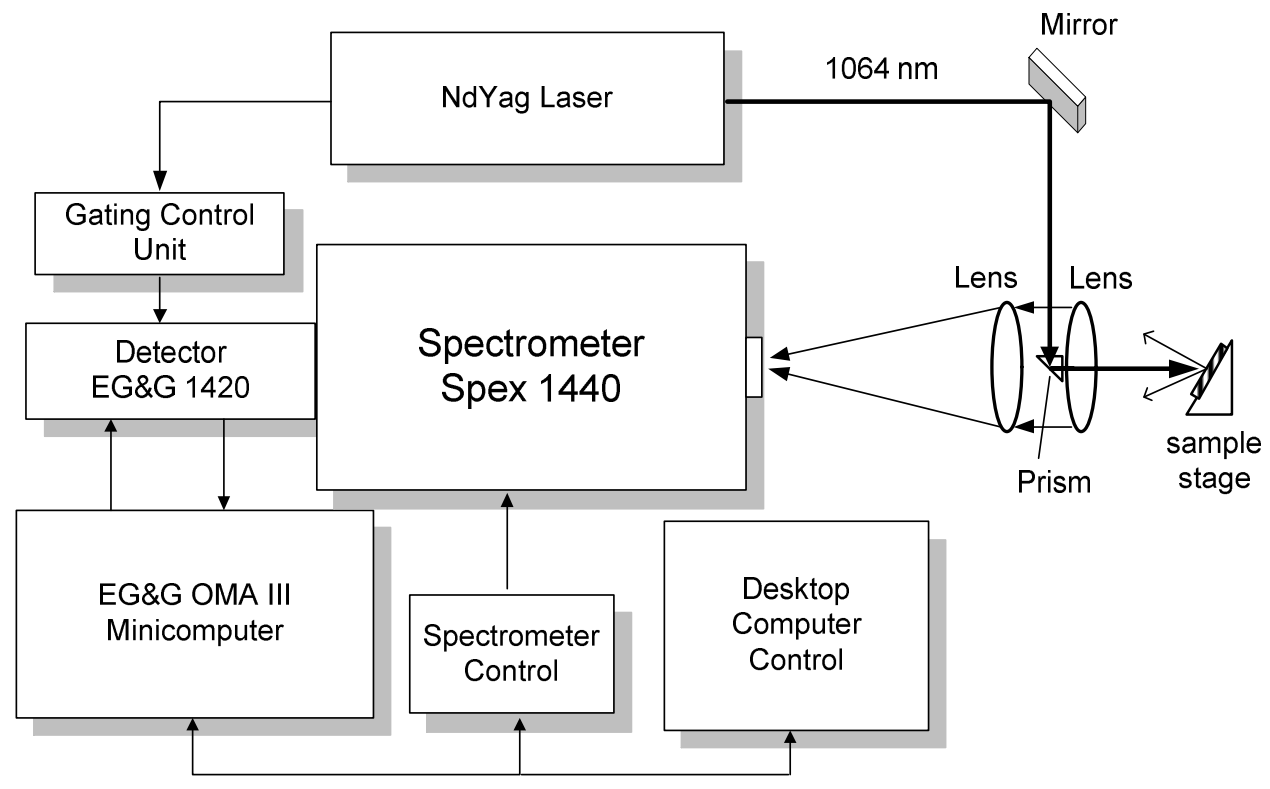

Fig. 1
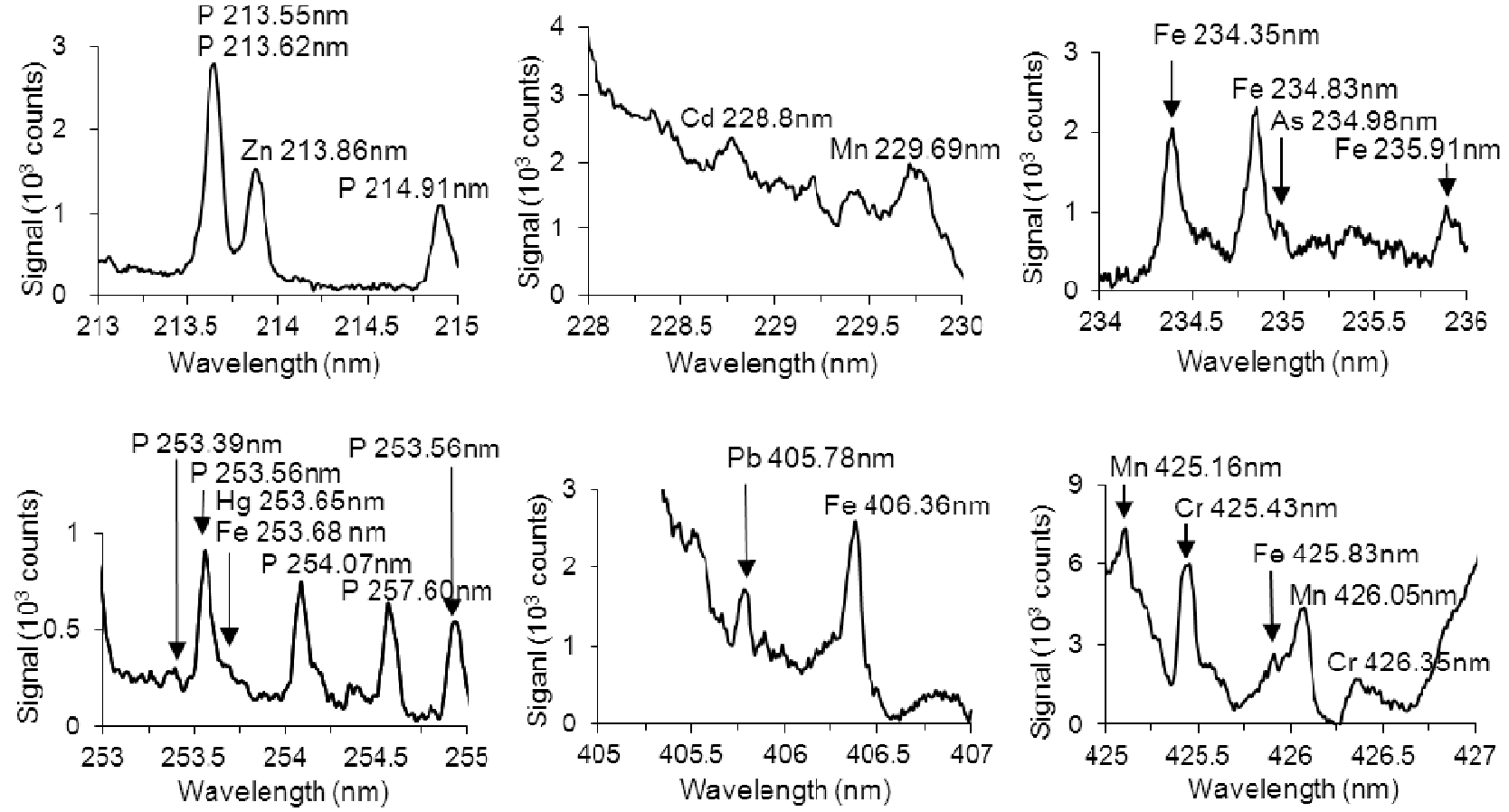

Fig. 2 

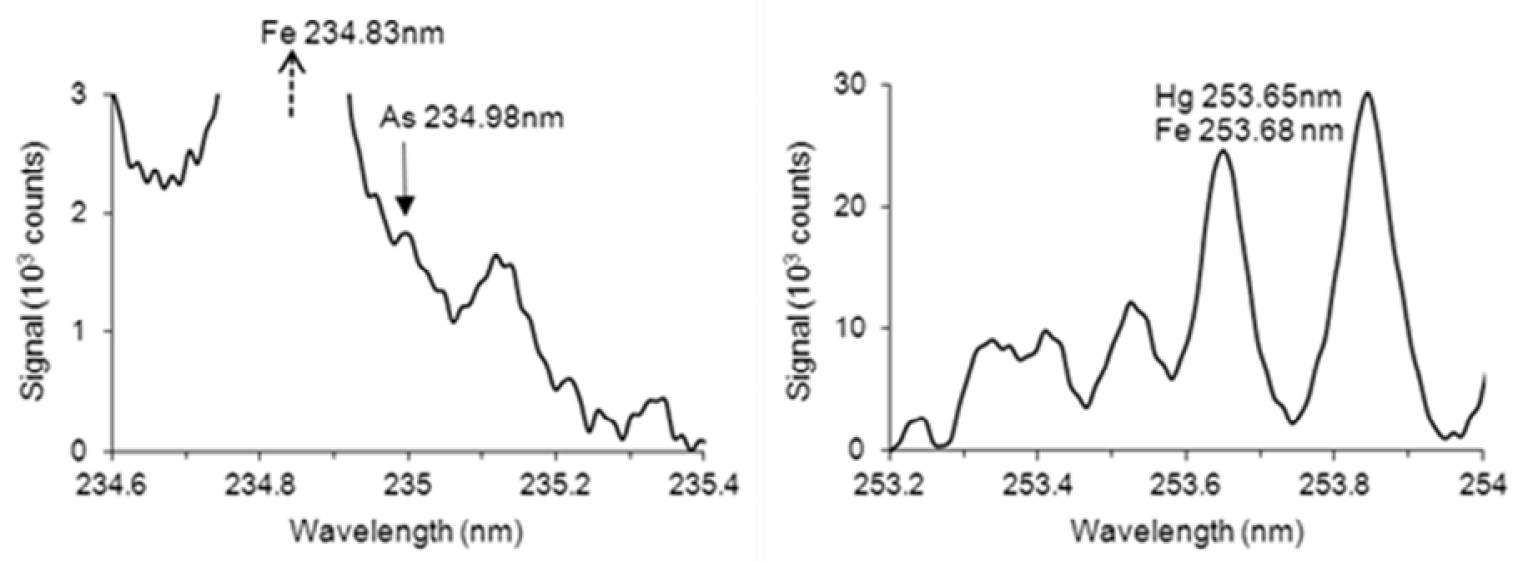

Fig. 3
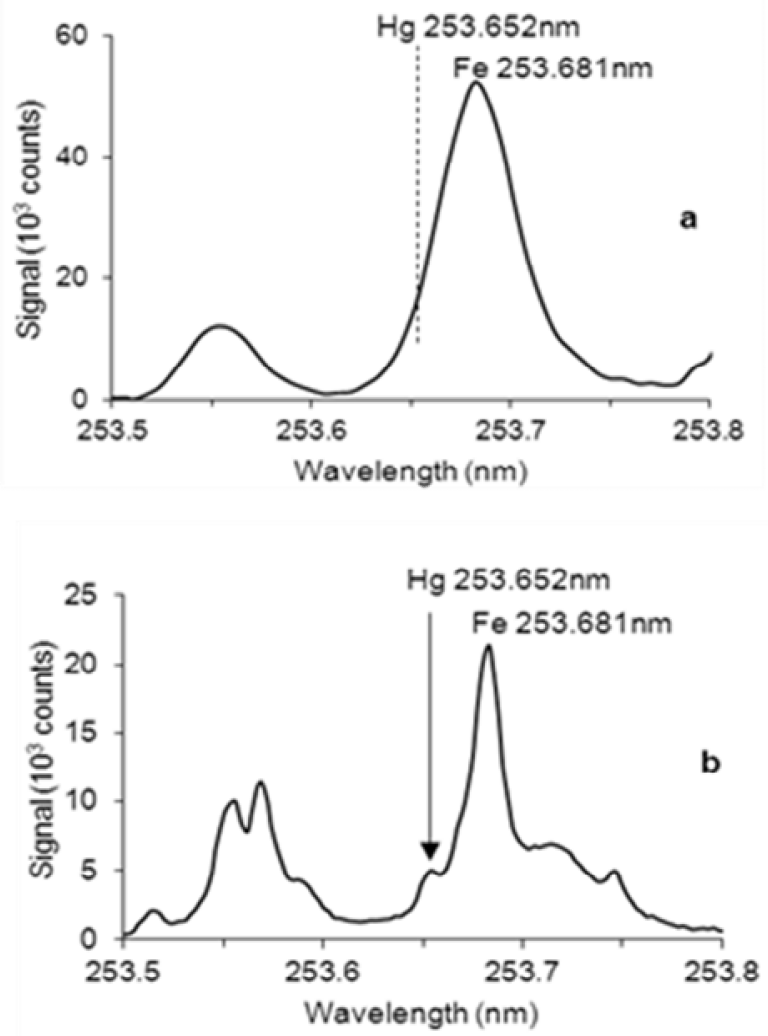

Fig. 4 

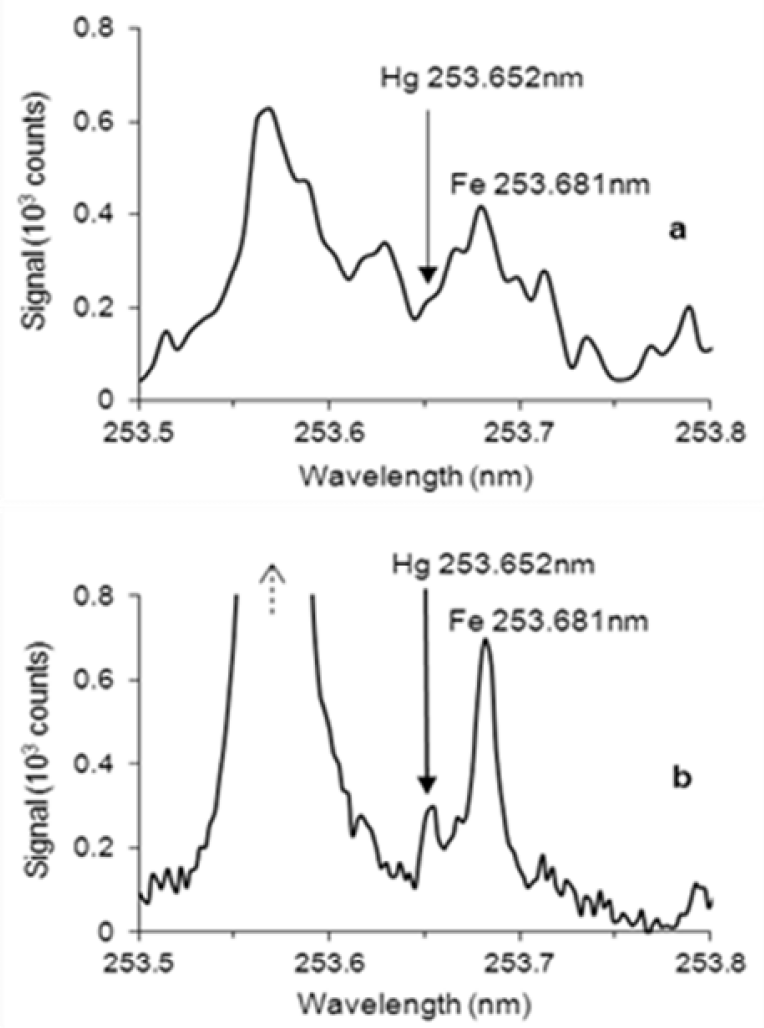

Fig. 5

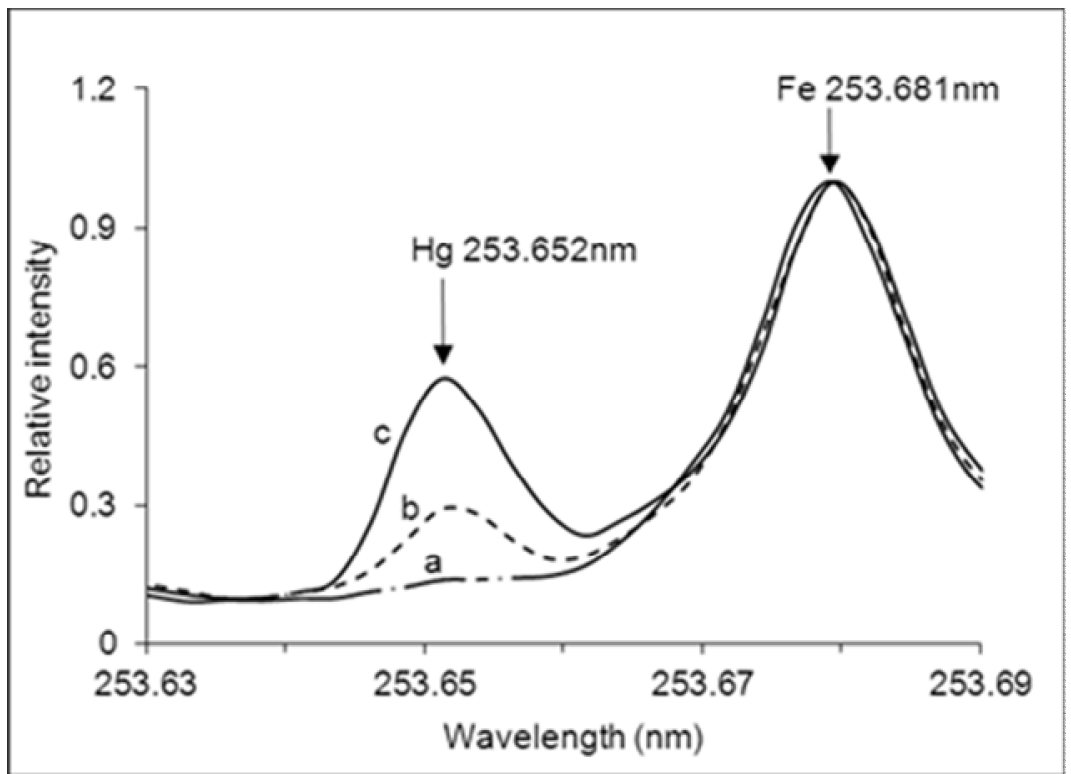

Fig. 6 


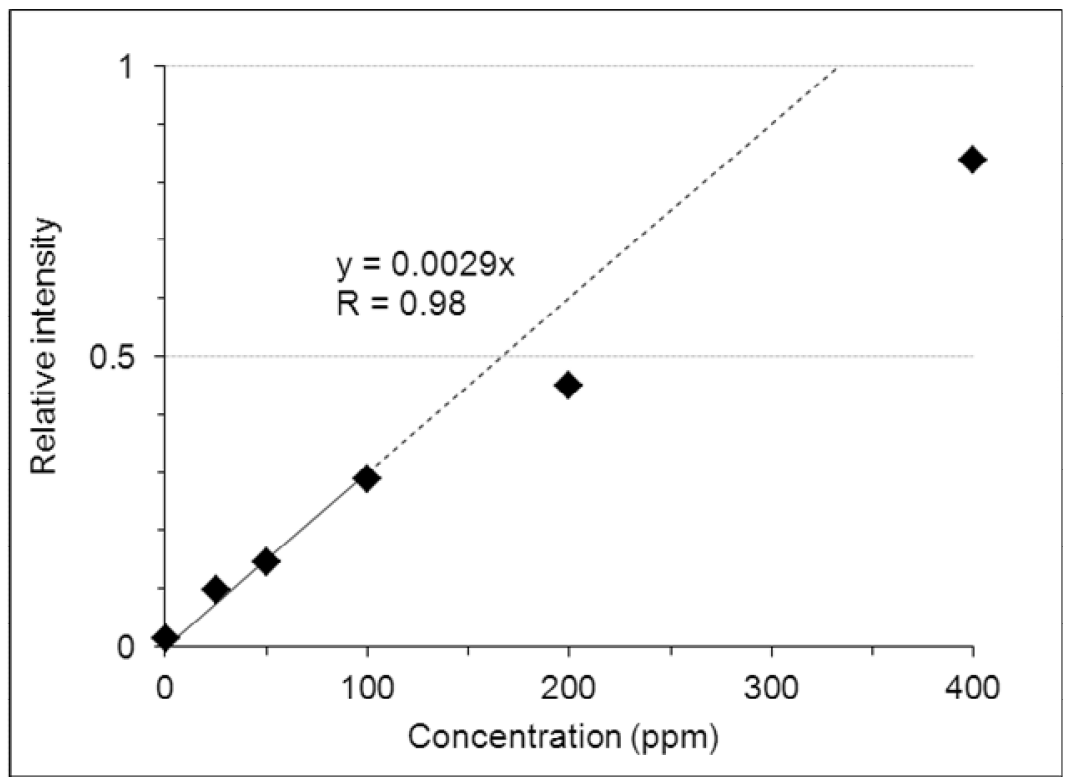

Fig. 7 